\title{
Erratum to: Isoform- and receptor-specific channel property of canonical transient receptor potential (TRPC)1/4 channels
}

\author{
Jinsung Kim • Misun Kwak • Jae-Pyo Jeon • Jongyun Myeong • Jinhong Wie • \\ Chansik Hong • Sung-Young Kim • Ju-Hong Jeon • Hyun Jin Kim • Insuk So
}

Published online: 3 December 2013

(C) Springer-Verlag Berlin Heidelberg 2013

\section{Erratum to: Pflugers Arch - Eur J Physiol DOI 10.1007/s00424-013-1332-y}

The authors would like to apologize for inadvertently using Fig. $1 \mathrm{~d}$ also for Fig. $5 \mathrm{~h}$ in the printed version of this paper.

The correct versions of Fig. 1d and Fig. $5 \mathrm{~h}$ are given below.

The online version of the original article can be found at http://dx.doi.org/ 10.1007/s00424-013-1332-y.

\section{J. Kim}

Department of Chemistry, Korea Advanced Institute of Science and

Technology, Daejeon 305-701, Republic of Korea

J. Kim $\cdot$ M. Kwak $\cdot$ J.-P. Jeon $\cdot$ J. Myeong $\cdot$ J. Wie $\cdot$ C. Hong $\cdot$

S.-Y. Kim $\cdot$ J.-H. Jeon $\cdot$ I. So $(\square)$

Department of Physiology, Seoul National University,

College of Medicine, Seoul 110-799, Republic of Korea

e-mail: insuk@plaza.snu.ac.kr

\section{H. J. Kim $(\bowtie)$}

Department of Physiology, Sungkyunkwan University, School of Medicine, Suwon 440-746, Republic of Korea e-mail:kimhyunjin@skku.edu

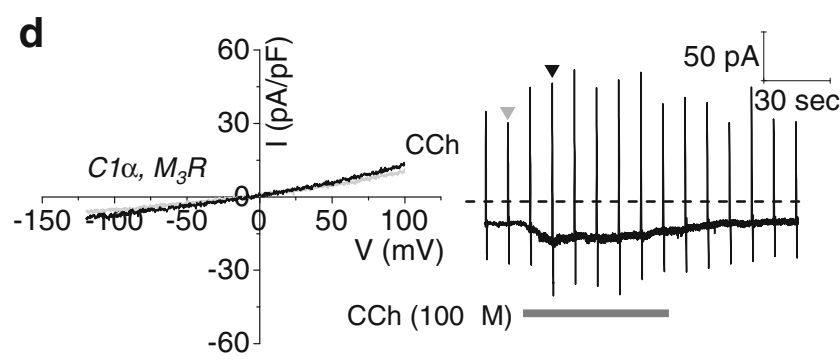

Fig. $1 \mathrm{G}_{\mathrm{q} / 11}$-coupled receptor stimulation and TRPC $1 \alpha$, TRPC $1 \alpha / 4$, TRPC4 channel activity. From a to $\mathbf{h}$, all left panels indicate $I-V$ relationship and right panels indicate corresponding current traces. Inset legends at upper left area of $I-V$ curves demonstrate heterologously expressed proteins. a, b In cells expressing M1 or M3 receptor, receptor stimulation by extracellular carbachol $(100 \mu \mathrm{M})$ did not induced significant whole-cell current. $\mathbf{c}, \mathbf{d}$ Cells expressing TRPC $1 \alpha$ channels showed indistinguishable current increment in response to M1 or M3 stimulation achieved by extracellular carbachol $(C C h, 100 \mu \mathrm{M})$. e, f Heteromeric TRPC $1 \alpha / 4$ channel showed typical outward-rectifying $I-V$ curves in response to M1 or M3 stimulation. $\mathbf{g}$, $\mathbf{h}$ Genuine TRPC4 homomeric currents (without co-expression of TRPC $1 \alpha$ ) in response to M1 or M3 receptor stimulation. i Left panel indicates summarized current density measured above and right panel indicates rectification factor $\left(\mid I_{+80 \mathrm{mV}} / \mathrm{I}\right.$ $-60 \mathrm{mV}$ ) analysis which was devised in order to show the sheer difference in $I-V$ curve signature. j Left panel compares double-rectifying $I-V$ curves of CCh-activated TRPC4 (light gray) and outward-rectifying I$V$ curves of CCh-activated TRPC1 $\alpha / 4$ (black). In order to quantify the order of outward rectification, another rectification factor $\rho$ (Rho) was devised which parameterizes two different slope conductance: $\rho \equiv \mid \mathrm{g}_{+}$ $10 \sim+50 \mathrm{mV}|/| \mathrm{g}_{-120 \sim-80 \mathrm{mV}} \mid$. Two different $I-V$ curves show stark difference in $\rho$ value 


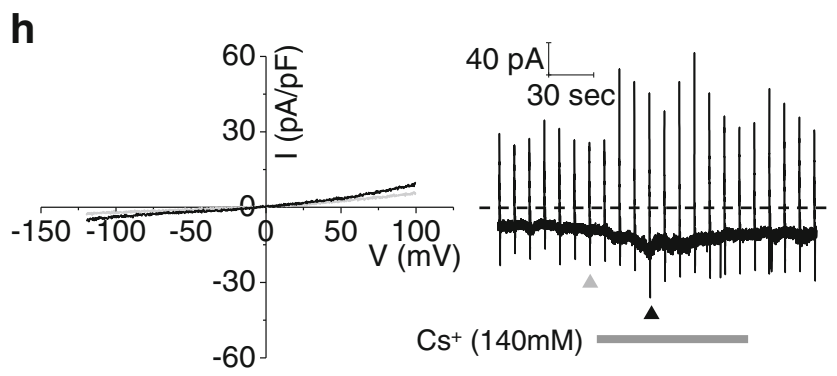

Fig. 5. TRPC $1 \alpha$, TRPC $1 \alpha / 4$, and TRPC4 channel activities in response to $\mathrm{G}_{\mathrm{i} / \mathrm{o}}$-coupled receptor (M2) stimulation and overexpression of $\mathrm{G} \alpha_{\mathrm{i} 2} \mathrm{Q}^{205}$ protein. a, b Genuine TRPC4 homomeric channel activity in response to $\mathrm{M} 2$ receptor stimulation and overexpression of $\mathrm{G} \alpha_{\mathrm{i} 2} \mathrm{Q} 205 \mathrm{~L}$ (intrinsic GTPase activity deficient mutant) protein. Extracellular carbachol $(C C h, 100 \mu \mathrm{M})$ and $\mathrm{Cs}^{+}$-rich solution $\left(\left[\mathrm{Cs}^{+}\right]_{\mathrm{o}}=140 \mathrm{mM}\right)$ was treated for each activation systems, respectively. TRPC4 channels activated by each condition showed typical double-rectifying $I-V$ curves. c, d TRPC $1 \alpha$ homomeric channel activities in response to $\mathrm{M} 2$ receptor and $\mathrm{G} \alpha_{\mathrm{i} 2}{ }^{\mathrm{Q} 205 \mathrm{~L}}$ protein. Neither M2 receptor stimulation nor $\mathrm{G} \alpha_{\mathrm{i} 2}$ Q205L protein activated TRPC $1 \alpha$ channels. e, f TRPC $1 \alpha / 4$ heteromeric channel activities in response to M2 receptor and $\mathrm{G} \alpha_{\mathrm{i} 2}$ Q205L protein. Each activation system was insufficient to induce TRPC $1 \alpha / 4$ channel currents but selectively activated TRPC4 homomeric channels. $\mathbf{g}, \mathbf{h}$ TRPC1 $\beta$ homomeric channel activities in response to M2 receptor and $\mathrm{G} \alpha_{\mathrm{i} 2}{ }^{\mathrm{Q} 205 \mathrm{~L}}$ protein. Both systems could not induce channel activation. $\mathbf{i}, \mathbf{j}$ TRPC1 $\beta / 4$ heteromeric channel activities in response toM2 receptor and $\mathrm{G} \alpha \alpha_{\mathrm{i} 2}{ }^{\mathrm{Q} 205 \mathrm{~L}}$ protein. Like TRPC $1 \alpha / 4$, TRPC1 $\beta / 4$ did not permit their activation to those stimulators but only TRPC4 did permit their activation 